\title{
Factors controlling upper tropospheric relative humidity
}

\author{
B. Kärcher and W. Haag \\ Deutsches Zentrum für Luft- und Raumfahrt (DLR), Institut für Physik der Atmosphäre, Oberpfaffenhofen, Germany
}

Received: 30 April 2003 - Revised: 8 July 2003 - Accepted: 22 July 2003 - Published: 19 March 2004

\begin{abstract}
Factors controlling the distribution of relative humidity in the absence of clouds are examined, with special emphasis on relative humidity over ice (RHI) under upper tropospheric and lower stratospheric conditions. Variations of temperature are the key determinant for the distribution of RHI, followed by variations of the water vapor mixing ratio. Multiple humidity modes, generated by mixing of different air masses, may contribute to the overall distribution of RHI, in particular below ice saturation. The fraction of air that is supersaturated with respect to ice is mainly determined by the distribution of temperature. The nucleation of ice in cirrus clouds determines the highest relative humdity that can be measured outside of cirrus clouds. While vertical air motion and ice microphysics determine the slope of the distributions of RHI, as shown in a separate study (Haag et al., 2003), clouds are not required to explain the main features of the distributions of RHI below the ice nucleation threshold.
\end{abstract}

Key words. Atmospheric composition and structure (pressure, density and temperature; troposphere - composition and chemistry; general or miscellaneous)

\section{Introduction}

Water is the only substance present in all three phases in the Earth's atmosphere: as vapor molecules in the gas phase, as cloud droplets in the liquid phase, and as ice crystals in the solid phase. The relative humidity over liquid water or ice indicates which phase is thermodynamically most stable at a given air temperature and pressure. The relative humidity, defined as the ratio of the water vapor pressure over the vapor pressure of water at liquid or ice saturation, plays a crucial role in cloud physics and atmospheric chemistry.

Clouds composed of water or ice particles form at certain relative humidities above liquid or ice saturation. Relative humidity controls the water activity (ratio between the water

Correspondence to: B. Kärcher

(bernd.kaercher@dlr.de) vapor pressures of a liquid solution and of pure water under the same conditions) of aerosol particles; the activity, in turn, determines the conditions under which cloud condensation nuclei form cloud droplets and supercooled liquid solutions form ice crystals (Koop et al., 2000). The rates of heterogeneous chemical reactions occurring in liquid aerosol particles are also strongly dependent on their water activity (Peter, 1997, and references therein).

This work studies the controlling factors of probability distributions of relative humidity in the atmosphere using simple analytical and statistical tools. The probability to find a given value for the relative humidity is controlled by air temperature and by the vapor pressure of water, which, in turn, are mainly affected by radiative processes, transport processes (advection, convection, turbulence, among other factors), and microphysical processes involving the formation and life cycle of clouds (condensation, latent heat release, precipitation, among others).

We do not intend to model radiative, microphysical, and transport processes that determine temperature and water vapor pressure. We rather prescribe distributions of temperature and water vapor mixing ratios and study the resulting distributions of relative humidity, ignoring the possibility of cloud formation. Although our findings are quite general in nature, we keep the focus on relative humidity over ice and the upper troposphere and lower stratosphere. It has been demonstrated that air in this region can be substantially supersaturated with respect to ice (Murphy et al., 1990; Heymsfield et al., 1998; Gierens et al., 1999; Ovarlez et al., 2000; Vay et al., 2000; Jensen et al., 2001; Spichtinger et al., 2002; Ovarlez et al., 2002), with important implications for the formation of cirrus clouds. The link between distributions of relative humidity over ice and the nucleation and initial growth of ice crystals in cirrus is explored in a separate study (Haag et al., 2003). 


\section{Basic ideas}

The saturation ratio over ice, $S$, is defined as

$S=p_{w} / p_{w s}(T)$.

Here, $p_{w}$ is the vapor pressure of water $\left(\mathrm{H}_{2} \mathrm{O}\right), T$ is the air temperature and $p_{w s}$ is the $\mathrm{H}_{2} \mathrm{O}$ saturation vapor pressure over ice, expressed as

$p_{w s}(T)=p_{v} \exp (-\theta / T)$.

The constants $p_{v} \simeq 3.445 \times 10^{10} \mathrm{hPa}$ and $\theta \simeq 6145 \mathrm{~K}$ are taken from laboratory measurements (Marti and Mauersberger, 1993). The saturation ratio over ice is fully equivalent to the relative humidity over ice, RHI, because, by definition, $\mathrm{RHI}=S \cdot 100 \%$. Equation (2) is applicable in the temperature range $170-250 \mathrm{~K}$ and represents a highly accurate fit of measured ice vapor pressures under cirrus and polar stratospheric cloud conditions.

We ask the fundamental question: Given probability distributions for $T$ and $p_{w}$, what are the resulting probability distributions for $S$ ? We briefly address the potential effects of clouds and precipitation on the distributions for $S$ at the end of Sect. 4.

Let us generalize $p_{w}$ or $T$ by $\phi$ and define the corresponding probability distribution $\Phi(\phi)$. Assuming for the moment that only one of these variables, $p_{w}$ or $T$, is changing, the answer to our question is obtained by

$\Psi(S)=\left|\phi^{\prime}(S)\right| \Phi[\phi(S)]$,

with the inverse function $\phi(S)$, its first derivative $\phi^{\prime}=d \phi / d S$, and the desired probability distribution for $S, \Psi(S)$. Equation (3) essentially states that the probability of finding a certain value $\phi$ within $\phi$ and $\phi+\Delta \phi$ is equal to the probability of finding $S(\phi)$ within the corresponding interval $S$ and $S+\Delta S$. We could generalize Eq. (3) to include the dependence of $\phi$ on two simultaneously changing variables $p_{w}$ or $T$, but we will not study such cases analytically.

The function $\Psi(S)$ can also be deduced numerically using a statistical approach. As we will discuss discrete distributions in Sect. 3, we briefly sketch how we proceed in this case. We make use of the random number generator ran 1 (Press et al., 1992, p.269f). This tool produces real numbers uniformly distributed between 0 and 1 , and is used to derive uniform probability functions for $T$ and $p_{w}$ by simple scaling. Using $\operatorname{ran} 1$ as the source of random numbers, we apply the transformation method described in Press et al. (1992, p.279f) to generate normally distributed values of $\phi$. The discrete distributions obtained in this way are inserted into Eq.(1), and the resulting values of $S$ are grouped into intervals, leading to discrete distribution functions of $S$. As demonstrated in Sect. 4, this approach is very useful in simulating distributions of relative humidity using real $T$ and $p_{w}$ data.

In principle, Eq. (3) allows us to compute the distribution of $S$ from any prescribed distribution function $\Phi(T)$. Two examples for normalized distributions of $T$ that will be employed in Sect. 3 are the uniform distribution

$\Phi_{u}(T)=\left\{\begin{array}{cl}\left(T_{\max }-T_{\min }\right)^{-1}: & T_{\min } \leq T \leq T_{\max } \\ 0: & \text { otherwise },\end{array}\right.$

and the Gaussian (normal) distribution

$\Phi_{n}(T)=\frac{1}{\sigma} \frac{1}{\sqrt{2 \pi}} \exp \left[-\frac{\left(T-T_{\mathrm{o}}\right)^{2}}{2 \sigma^{2}}\right]$.

with the mean temperature $T_{\mathrm{o}}$ and the standard deviation $\sigma$.

The box distribution has no evident atmospheric relevance and is discussed below only for illustration purposes. According to the central limit theorem (Jeffreys, 1948), normal distributions approximate the peak region (the region containing most of the chance to find events) of any stochastic distribution very well for a sufficiently large ensemble of independent events.

In their trajectory study of water vapor transport in the tropical tropopause layer, Gettelman et al. (2002) have added normally distributed variations of $T$ with $\sigma=2 \mathrm{~K}$ to synopticscale temperature fields, to account for variations caused by spatially and temporally unresolved gravity waves. A similar approach was taken by Tabazadeh et al. (1996) to study the formation of polar stratospheric clouds.

Even if temperature distributions approach a Gaussian shape in the peak region, they may exhibit a certain degree of skewness and show a different functional behavior for the very rare events in the wings of the distribution. For instance, it is known that mesoscale temperature fluctuation amplitudes are characterized by normal distributions close to the peak, but fall off much less rapidly in the high-amplitude tails (Bacmeister et al., 1999). Lorentzian distributions of $T$ can be used to fit instantaneous fluctuations of atmospheric temperatures at certain times over large $\left(\sim 10^{5} \mathrm{~km}^{2}\right)$ spatial scales (Gierens et al., 1997). This type of short-term variations between RHI data taken from different spatial regions is also discussed in the SPARC Water Vapor Assessment (2000).

In Sect. 4.1 we study the applicability of Gaussian distributions of $T$ to a large, random sample of atmospheric temperatures gathered on a regional scale in four weeks of airborne in situ measurements. This approach differs from studying temperature fluctuations in moving (Lagrangian) air parcels on the mesoscale (see Sect. 4.3 for more details). In summary, we are fully aware about the limitations assuming Gaussian temperature distributions. We do not claim this assumption to hold in all conceivable cases, but show that assumed normal distributions of $T$ can reproduce all essential features of the resulting distributions of $S$ below the cirrus cloud formation threshold. 


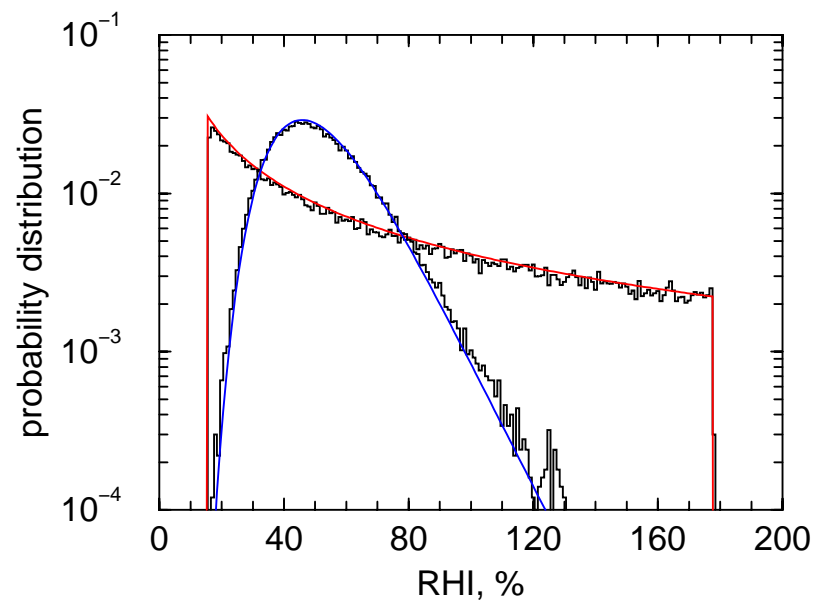

Fig. 1. Probability distributions of RHI obtained with a uniform distribution of $T$ in the interval 215-235 $\mathrm{K}$ (red curve) and a normal distribution with $T_{\mathrm{O}}=225 \mathrm{~K}$ and $\sigma=2.5 \mathrm{~K}$ (blue curve). The stair steps depict the corresponding discrete distributions obtained with random samples of $T$, using 50,000 individual random numbers binned into $1 \%$ intervals.

\section{Applications}

\subsection{Varying the temperature $T$}

We study the probability distributions for $S$ in the case of pure temperature variations. In this case, we have $\phi=T$, and $\phi^{\prime}(S)$ reads

$T^{\prime}(S)=-\theta /\left[S \ln ^{2}(S / \alpha)\right], \quad \alpha=p_{w} / p_{v}$,

where we have used $T(S)=\theta / \ln (S / \alpha)$ obtained by inverting Eq. (2) and inserting the result into Eq. (1).

In the case of a uniform distribution, we simply have

$\Phi_{u}[T(S)]=\left\{\begin{array}{cl}\left(T_{\max }-T_{\min }\right)^{-1}: & S_{\max } \geq S \geq S_{\min } \\ 0 \quad: & \text { otherwise }\end{array} ;\right.$

inserting this expression into Eq. (3) yields

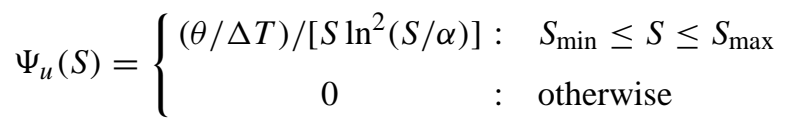

with $\Delta T=T_{\max }-T_{\min }$.

In the case of a normal distribution, we obtain

$\Phi_{n}[T(S)]=\frac{1}{\sigma} \frac{1}{\sqrt{2 \pi}} \exp \left\{-\beta\left[\frac{1}{\ln (S / \alpha)}-\frac{1}{\ln \left(S_{\mathrm{o}} / \alpha\right)}\right]^{2}\right\}$,

with $S_{0}=p_{w} / p_{w s}\left(T_{0}\right)$ and $\beta=1 /\left(2 \Sigma^{2}\right)$, where we have defined the scaled standard deviation $\Sigma=\sigma / \theta$. Inserting this expression into Eq. (3) yields

$\Psi_{n}(S)=\frac{1 /(\Sigma \sqrt{2 \pi})}{S \ln ^{2}(S / \alpha)} \exp \left\{-\beta\left[\frac{1}{\ln (S / \alpha)}-\frac{1}{\ln \left(S_{\mathrm{o}} / \alpha\right)}\right]^{2}\right\}$

The probability distributions $\Psi_{u}(S)$ and $\Psi_{n}(S)$ from Eqs. (8) and (10), respectively, are shown in Fig. 1 as solid

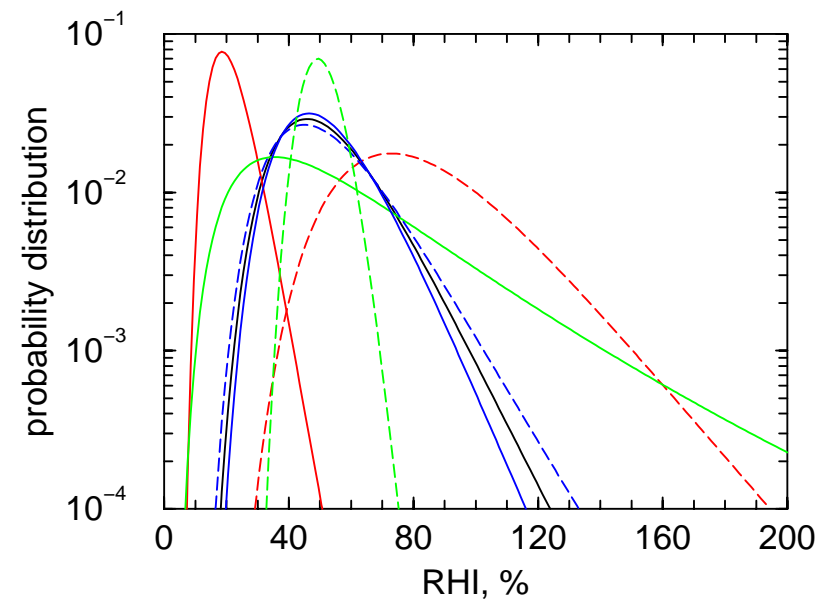

Fig. 2. Probability distributions of RHI obtained with normal distributions of $T$. The black curve is equal to the baseline case shown as the blue curve in Fig. 1. Red, green, and blue curves are obtained by varying $\mathrm{RHI}_{\mathrm{o}}, \sigma$, and $T_{\mathrm{O}}$, respectively. See text for details.

curves. Also shown as stair steps are the same distribution functions obtained with random samples of $T$. We have chosen $p_{w}$ such that $S_{0}=0.5$, or $\mathrm{RHI}_{0}=50 \%$. Note that the statistical uncertainty increases significantly towards very low and very high relative humidities (rare events with probabilities $\left.\ll 10^{-3}\right)$ in the case of the discrete distributions.

We study the distributions of RHI resulting from Gaussian distributions of $T$ in more detail with the help of Fig. 2. The black curve represents the baseline case already discussed above, see blue curve in Fig. 1, with the values $T_{0}=225 \mathrm{~K}$, $\sigma=2.5 \mathrm{~K}$, and $\mathrm{RHI}_{\mathrm{o}}=50 \%$.

The blue curves are obtained by prescribing a mean temperature $T_{\mathrm{o}}$ of $235 \mathrm{~K}$ (solid) and $215 \mathrm{~K}$ (dashed), keeping $\mathrm{RHI}_{\mathrm{O}}$ unchanged. This has only little influence on the distribution of RHI.

Varying the width $\sigma$ of the temperature distribution changes the distribution of RHI more significantly. When increasing $\sigma$ to $5 \mathrm{~K}$ (solid green curve), the probability to find ice supersaturation increases dramatically, but also lower values are present compared to the baseline case. The maximum shifts to the left. When decreasing it to $\sigma=1 \mathrm{~K}$ (dashed green curve), the distribution becomes much narrower, and is sharply peaked around $\mathrm{RHI}_{0}=50 \%$. This behavior is caused by the fact that the total probability given by the area under the curves is a conserved quantity.

Decreasing or increasing the mean value of $\mathrm{RHI}_{\mathrm{O}}$ to $80 \%$ (dashed red curve) and 20\% (solid red curve) has similar effects; the maximum shifts to the right (left) and takes on lower (higher) values. The distribution flattens and high values of RHI are obtained when $\mathrm{RHI}_{\mathrm{O}}$ rises. Note that the maximum of $\Psi$ is not always located at $\mathrm{RHI}_{0}$, except in the limit $\sigma \rightarrow 0$.

The choices of $\sigma=1-5 \mathrm{~K}$ are typical for regional-scale variations of temperatures, as demonstrated in Sect. 4.1. In summary, it is easy to create high probabilities to find 


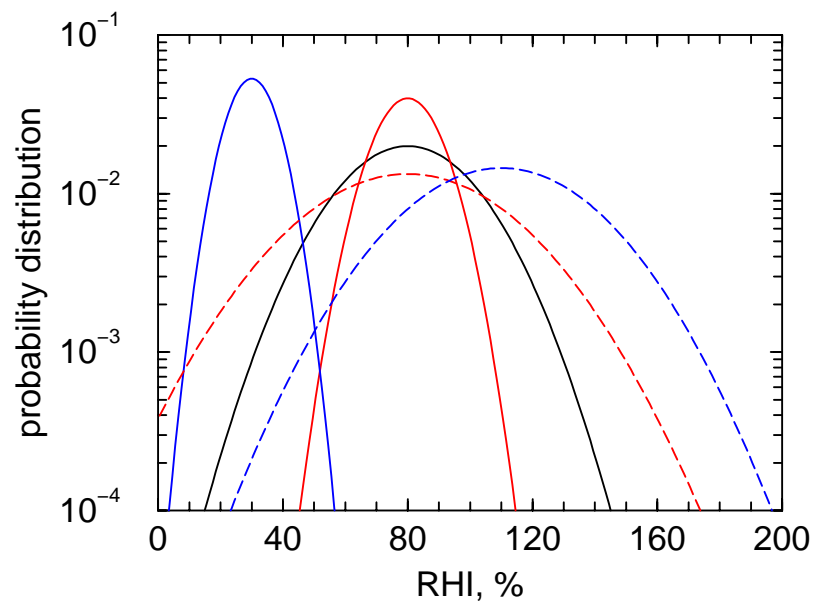

Fig. 3. Probability distributions of RHI obtained with normal distributions of the $\mathrm{H}_{2} \mathrm{O}$ mixing ratio $\chi$ at constant temperature. The black and colored curves show analytical solutions. The baseline case (black curve) assumes $T=225 \mathrm{~K}, p=250 \mathrm{hPa}, \chi_{\mathrm{o}}=160 \mathrm{ppm}$, leading to $\mathrm{RHI}_{\mathrm{o}}=80 \%$, and $\sigma_{w}=80 \mathrm{ppm}$. Red (blue) curves are obtained by changing $\sigma_{w}\left(\mathrm{RHI}_{\mathrm{O}}\right)$. See text for details.

supersaturation by such variations of air temperature, even if the mean $\mathrm{RHI}_{\mathrm{o}}$ is well below $100 \%$.

\subsection{Effect of adiabatic changes of $p_{w}$}

In the above discussion, we have ignored the changes of $p_{w}$ associated with changes of $T$ in an adiabatically rising or sinking air parcel. This leads to a $T$-dependence of $p_{w}$ of the form

$p_{w}=p_{w}\left(T_{\mathrm{o}}\right)\left(T / T_{\mathrm{o}}\right)^{\kappa}$,

with $\kappa \simeq 3.5$ for dry air. This expression must be inserted into Eq. (1), whereby the inverse function $T(S)$ needed to write the distribution function of $S$ is not available in a closed form in this case.

An inspection of the distributions of RHI, including adiabatic changes of $p_{w}$ (not shown), reveals that the distributions that include these changes are slightly narrower than those evaluated with constant $p_{w}$, and that the peak probabilities are therefore slighty higher. To be more quantitative, the changes of the solid distributions given in Fig. 2 above a probability value of $\sim 8 \times 10^{-3}$ caused by adiabatic motions are almost invisible. The reason for this very moderate influence lies in the fact that changes of $T$ via $p_{w}$ according to Eq.(11) have less effect on $S$ than changes of $T$ via $p_{w s}$, because $p_{w s}$ depends exponentially on $T$.

\subsection{Varying the $\mathrm{H}_{2} \mathrm{O}$ mixing ratio $\chi$}

It is instructive to study the probability distributions for $S$ in the case of pure variations of the water vapor mixing ratio $\chi=p_{w} / p$ ( $p$ denotes the air pressure). We rewrite Eq. (1) in the form

$S=\chi p / p_{w s}(T)$.
In this case we have $\phi=\chi$, and $\phi^{\prime}(S)$ reads

$\chi^{\prime}(S)=1 / \delta, \quad \delta=p / p_{w s}(T)$.

Because $\chi^{\prime}(S)$ is a constant, $\Psi(S)$ will be strictly proportional to $\Phi(\chi)$. Normally distributed $\mathrm{H}_{2} \mathrm{O}$ mixing ratios are of the form

$\Phi_{n}(\chi)=\frac{1}{\sigma_{w}} \frac{1}{\sqrt{2 \pi}} \exp \left[-\frac{\left(\chi-\chi_{\mathrm{o}}\right)^{2}}{2 \sigma_{w}^{2}}\right]$,

where $\chi_{\mathrm{o}}=p_{w o} / p$. The resulting distribution of $S$ reads

$\Psi_{n}(S)=\frac{1}{\Sigma_{w}} \frac{1}{\sqrt{2 \pi}} \exp \left[-\beta_{w}\left(S-S_{\mathrm{o}}\right)^{2}\right]$,

with the scaled standard deviation $\Sigma_{w}=\delta \sigma_{w}$ and $\beta_{w}=1 /\left(2 \Sigma_{w}^{2}\right)$. The distribution width $\Sigma_{w}$ does not depend on $T$ when $\sigma_{w} \propto \chi_{\mathrm{o}}$; in such practically relevant cases, the dependences of $\chi_{\mathrm{o}}$ and $\delta$ on $p_{w s}\left(\chi_{\mathrm{o}} \propto p_{w s}, \delta \propto 1 / p_{w s}\right)$ cancel each other in $\Sigma_{w}$.

Figure 3 depicts the Gaussian solutions for the distribution of RHI from Eq. (15) when Eq. (14) is prescribed for $T=225 \mathrm{~K}$ and $p=250 \mathrm{hPa}$ and with different sets of parameters. The baseline case (black curve) assumes $\sigma_{w}=0.5 \chi_{\mathrm{o}}$; the choice $\chi_{\mathrm{o}}=160 \mathrm{ppm}$ yields $\mathrm{RHI}_{\mathrm{o}}=80 \%$.

Upon increasing or decreasing the standard deviation of $\chi$ to $\sigma_{w}=0.75 \chi_{\mathrm{o}}$ (dashed red curve) or $\sigma_{w}=0.25 \chi_{\mathrm{o}}$ (red curve), the distributions of RHI become broader or narrower, respectively. Shifting $\mathrm{RHI}_{\mathrm{O}}$ to $30 \%$ (blue curve) or $110 \%$ (dashed blue curve) using $\sigma_{w}=0.5 \chi_{\mathrm{o}}$ leads to narrower and broader distributions, respectively. In contrast to Fig. 2, the maxima are always located exactly at $\mathrm{RHI}_{0}$.

The choices $\sigma_{w}=(0.25-0.75) \chi_{\mathrm{o}}$ are likely upper limits, as individual humidity modes contributing to real distributions of $\chi$ are likely characterized by smaller variances, see Sect. 4.1. In summary, if the variation of $\chi$ is the only reason for changes of $S$, the distributions of $\chi$ must be centered above ice saturation and must exhibit quite a large variance $\sigma_{w}$, in order to create a notable probability to find data points above ice saturation.

\subsection{Effect of combined changes of $p_{w}$ and $T$}

Let us assume for the moment that temperature and water vapor mixing ratios are independent variables. We vary them simultaneously to examine their combined effect on the distribution of RHI. As a closed-form solution is not available in this case, we study results obtained with the statistical approach using normal distributions of $T$ and $\chi$, with the baseline values $T_{\mathrm{o}}=225 \mathrm{~K}, \sigma=2.5 \mathrm{~K}, \mathrm{RHI}_{\mathrm{o}}=50 \%$, and $\sigma_{w}=0.5 \chi_{\mathrm{o}}$.

From the above presentation of results we may expect that combined variations of $T$ and $\chi$ lead to broader distributions of RHI than pure variations of either $T$ or $\chi$. In fact, this is seen in Fig. 4. The solid distributions take into account a normal distribution of $T$ and adiabatic changes, as discussed in Sect. 3.2. The corresponding dashed distributions additionally include a normal distribution of $\chi$. 


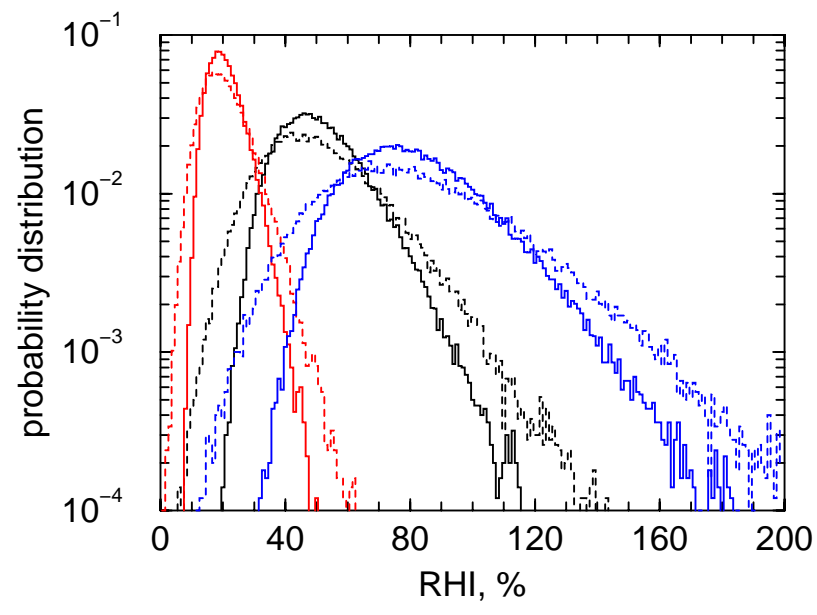

Fig. 4. Probability distributions of RHI obtained with normal distributions of $T$ and $\chi$, assuming that both quantities are uncorrelated. The solid black/red/blue distributions here include variations of $T$ and adiabatic pressure changes. We use the baseline values $T_{\mathrm{O}}=225 \mathrm{~K}, \sigma=2.5 \mathrm{~K}$, and $\mathrm{RHI}_{\mathrm{O}}=50 \%$. The dashed distributions additionally include variations of $\chi$, with the baseline value $\sigma_{w}=0.5 \chi_{\mathrm{o}}$. Red and blue distributions assume $\mathrm{RHI}_{\mathrm{O}}=20 \%$ and $\mathrm{RHI}_{\mathrm{O}}=80 \%$, respectively.

We find that the assumed variations of $\chi$ overcompensate the effect of adiabatic pressure changes and lead to an overall broadening of the distribution of RHI. The dominant features of the distributions of RHI, however, are still caused by the temperature variability, as illustrated in Fig. 2.

\section{Discussion and Summary of Results}

\subsection{Applying the results to in situ observations}

As an application, we discuss temperature and water vapor data taken during the INCA (Interhemispheric Differences in Cirrus Properties From Anthropogenic Emissions) field experiments. Measurements were carried out in the Northern Hemisphere $(\mathrm{NH})$ over the eastern North Atlantic region, starting from Prestwick, Scotland, and in the Southern Hemisphere (SH) west of south Chile, starting from Punta Arenas. Data have been taken in a period of four weeks in the regions $52-55^{\circ} \mathrm{N}$ and S. Details of the aircraft measurements of relative humidity are discussed elsewhere (Ovarlez et al., 2002).

Here, we employed data taken below $235 \mathrm{~K}$. Cirrus formation could have resulted in a feedback on temperatures taken inside clouds due to latent heat effects, but this introduces only a small, if any, effect, as we find no systematic difference between in-cloud and out-of-cloud temperature distributions.

To derive probability distributions of $S$ where the effect of clouds are eliminated, we have first calculated the means and variances directly from the measured temperatures. The dis-

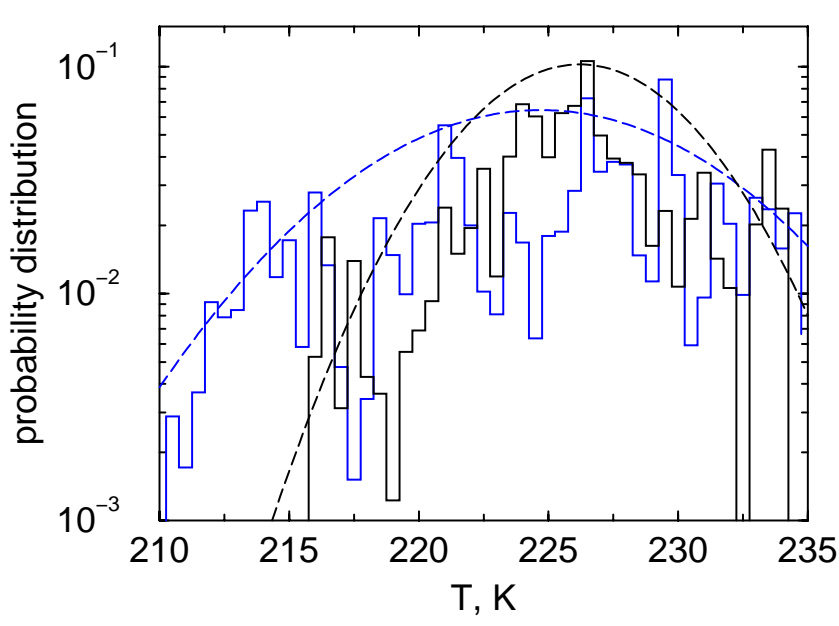

Fig. 5. Probability distributions of $T$ measured during the INCA experiment (Prestwick, black curves; Punta Arenas, blue curves). The stair steps represent the discretized in situ data. The number of data points is 59,444 (Punta) and 50,412 (Prestwick). The dashed curves are normal distributions evaluated with mean temperatures and standard variations obtained from the respective data sets.

tributions of $T$ measured in both campaigns shown in Fig. 5 were relatively similar, with mean values

$\bar{T}=\frac{1}{N} \sum_{j=1}^{N} T_{j}$,

of $224.7 \mathrm{~K}(\mathrm{SH})$ and $226.2 \mathrm{~K}(\mathrm{NH})$ and standard deviations

$\sigma_{T}=\sqrt{\frac{1}{N-1} \sum_{j=1}^{N}\left(T_{j}-\bar{T}\right)^{2}}$.

of $6.2 \mathrm{~K}(\mathrm{SH})$ and $3.9 \mathrm{~K}(\mathrm{NH})$. Besides the original measurements plotted as discrete distribution functions, Fig. 5 depicts normal distributions evaluated with the above mean values and standard deviations, demonstrating that the key features of the temperature observations are captured using this simple approach. We discuss the differences between the resulting distributions of RHI obtained directly with the data and with the Gaussian fits below after the brief description of the INCA $\mathrm{H}_{2} \mathrm{O}$ data.

The distributions of $\chi$ observed during the $\mathrm{NH}$ (black curve) and SH (blue curves) campaigns are shown in the top panel of Fig. 6. There seem to be several modes contributing to the overall distribution in both cases, most notably very dry ones centered around $15 \mathrm{ppm}(\mathrm{NH})$ and $25 \mathrm{ppm}$ (SH), likely to be of stratospheric origin. Other modes appear around $50 \mathrm{ppm}, 130 \mathrm{ppm}$, and $250 \mathrm{ppm}(\mathrm{NH})$, and $85 \mathrm{ppm}, 175 \mathrm{ppm}$, and $225 \mathrm{ppm}(\mathrm{SH})$. Note that while the overall distributions of $\chi$ have rather large variances $(\mathrm{NH}$ : $\sigma_{w}=85.1 \mathrm{ppm}=0.74 \chi_{\mathrm{o}}$; SH: $\sigma_{w}=76.8 \mathrm{ppm}=0.66 \chi_{\mathrm{o}}$ ), the variances of the individual dry and humid modes that can be observed in Fig. 6 (top panel) are considerably smaller.

The bottom panel is a scatter plot of the $T$ and $\chi$ data, suggesting the existence of correlations between the two data 

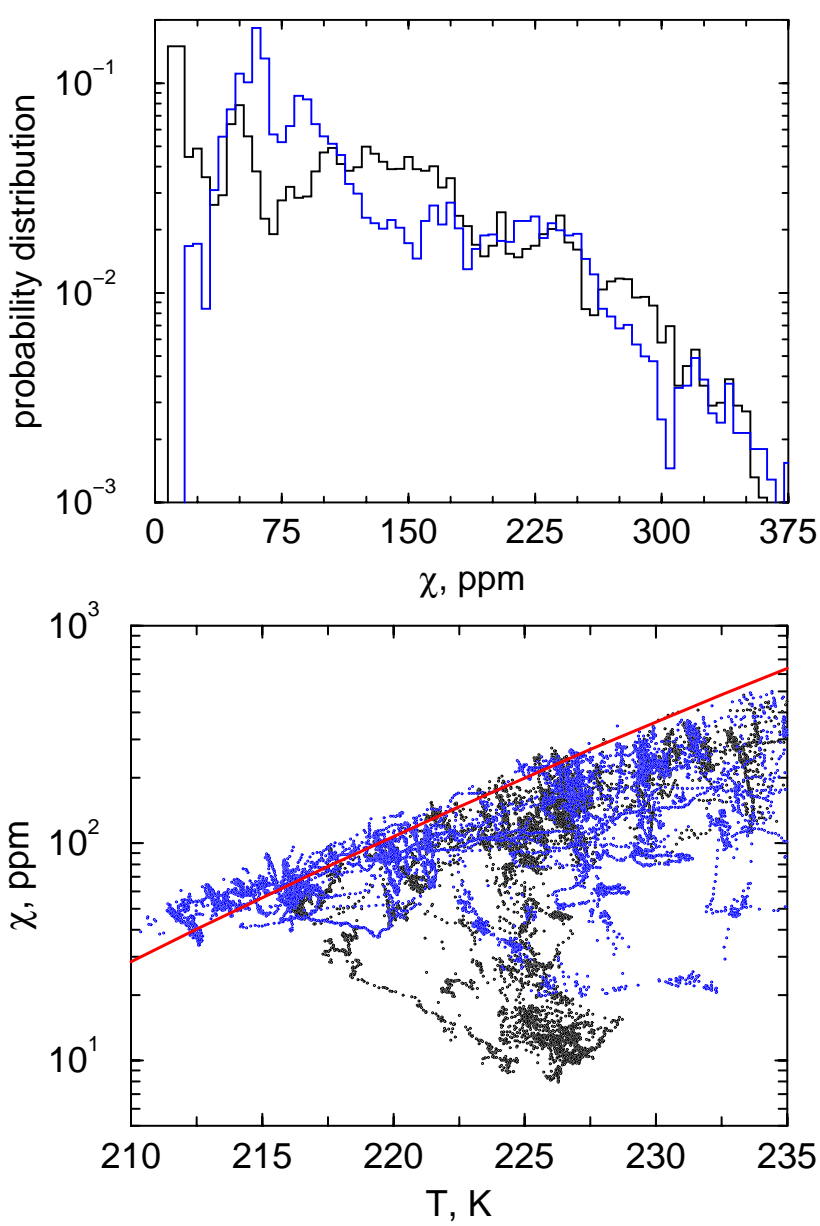

Fig. 6. Top panel: Probability distributions of $\chi$ measured during the INCA experiment (Prestwick, black curves; Punta Arenas, blue curves). The stair steps represent the discretized in situ data. Bottom panel: Scatter plot of water vapor mixing ratios and temperatures observed during INCA using the same color coding. The red curve marks the frost point $\mathrm{H}_{2} \mathrm{O}$ mixing ratios, evaluated for a constant pressure of $250 \mathrm{hPa}$.

sets generated in part by the aircraft flight patterns. The red curve marks the frost point values of $\chi$, evaluated at a constant air pressure of $250 \mathrm{hPa}$, close to the typical measurement altitude. An increasing number of data points lie above the red line when the temperature decreases, indicating a substantial barrier to the nucleation of cirrus clouds (Ström et al., 2003; Haag et al., 2003). The steady increase of the barrier with decreasing $T$ is consistent with the laboratory measurements reported by Koop et al. (2000).

We have calculated the probability distributions of RHI using the measured temperatures displayed in Fig. 5 under the assumption of constant water vapor mixing ratios (fixed at the mean observed relative humidities), because of the possibilty that $T$ and $\chi$ data are correlated. The mean values of $\mathrm{RHI}_{\mathrm{O}}$ used are $\sim 75 \%(\mathrm{NH})$ and $\sim 85 \%$ (SH) (Ovarlez et al., 2002). The results are shown as black distributions in Fig. 7. Adiabatic pressure changes were included in the calculations. The distributions extend well into the ice-supersaturated re- gions, with the SH data exhibiting somewhat higher probabilities to find supersaturation than the $\mathrm{NH}$ data.

To produce the red distributions in Fig. 7, we have used normal distributions of $T$ with the parameters $T_{\mathrm{o}}=\bar{T}$ and $\sigma=\sigma_{T}$ inferred from the measurements (see above), again including adiabatic pressure changes. This already leads to a good fit for the NH data, but is too broad in the case of the SH data. The reason is that the measured distributions of $T$ are not perfect Gaussians, with greater discrepancies in the SH case (Fig. 5), and that we have ignored possible variations of $\chi$. The fits could be improved by further allowing $\chi$ to vary, which tends to increase the slope of the RHI distributions above ice saturation (recall Fig. 4); however, due to the lack of knowledge to which degree $T$ and $\chi$ data are correlated, we do not attempt to combine the two data sets.

The blue distributions correspond to the observed RHI statistics, evaluated with the in situ measurements of RHI outside of cirrus clouds, as described in Haag et al. (2003). In both the $\mathrm{NH}$ and $\mathrm{SH}$ distributions, two modes appear centered at low RHI and near ice saturation, respectively. The dry modes with $\mathrm{RHI}_{\mathrm{o}} \simeq 5-10 \%$ are likely to be of stratospheric origin and have little influence on the spectra above saturation, as they fall off very rapidly already well below saturation. We recall that these dry modes are seen in the $\chi$-distributions displayed in Fig. 6 (top panel). They are not included in the calculated (red and black) distributions shown in Fig. 7.

In the NH case, the humid mode is very well captured by the simple model result (black solid curve). This is a remarkable feature, as the observed temperatures do not very closely follow the Gaussian fit. Nevertheless, assuming that the measured temperatures follow a normal distribution with mean values and standard deviation derived from the measurements and fixing the water mixing ratios with the observed mean relative humidities suffices to predict quite realistic distributions of RHI, compare the black and red humid modes in Fig. 7.

The key difference occurs above $\sim 130 \%$, where no NH data are found outside of clouds. This difference is attributed to cirrus formation (see Haag et al., 2003 for a comprehensive discussion and Ström et al., 2003 for an alternative explanation); here it is sufficient to state that the black distributions generated without taking into account ice nucleation are broad enough to produce the large supersaturations required for cirrus formation. In the SH case, the observed humid mode is centered at higher values of RHI, closer to the observed mean value of $\mathrm{RHI}_{\mathrm{O}} \simeq 85 \%$. The conclusion about the absence of SH data above $\sim 155 \%$ in the blue distribution relative to the black distribution is analogous to the $\mathrm{NH}$ case.

\subsection{Relating the results to previous work}

The first distributions of water vapor mixing ratios and ice saturation mixing ratios from airborne measurements in the tropopause region over Stavanger, Norway and Punta Arenas, Chile, have been discussed by Murphy et al. (1990). The authors focused on the interplay between temperature 

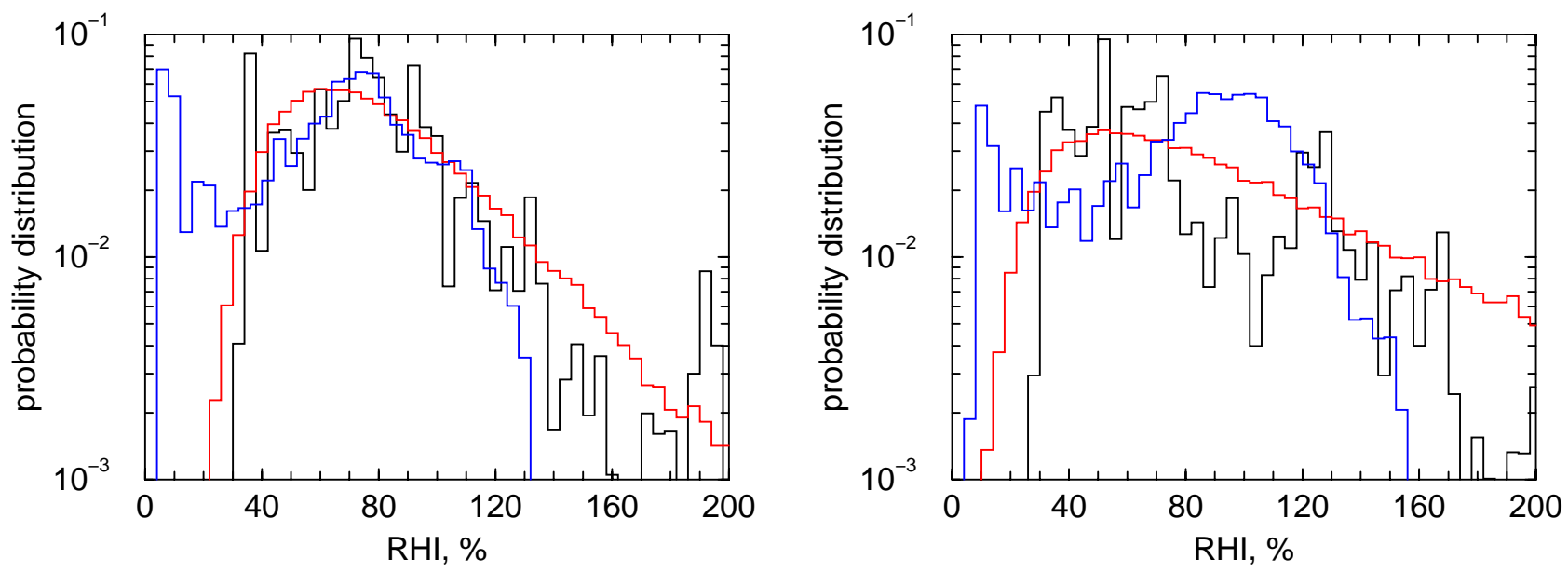

Fig. 7. Probability distributions of RHI (black curves) obtained with air temperatures measured during INCA (Prestwick, left panel; Punta Arenas, right panel). Red distributions are results from the statistical approach using Gaussian fits to the observed distributions of $T$ with mean and standard deviation derived from the measurements (see Fig. 5), and observed mean relative humidities over ice (NH: 75\%; SH: 85\%) taken from Ovarlez et al. (2002). Blue distributions are generated directly from the INCA RHI data taken outside of cirrus clouds (Haag et al., 2003).

and abundance of water vapor to explain the shape of the observed distributions.

A larger data set from the MOZAIC (Measurement of Ozone on Airbus in-service Aircraft) measurements has been analyzed by Gierens et al. (1999). The set comprises three years of data in the pressure range 175-275 $\mathrm{hPa}$, mainly taken above the North Atlantic along major commercial flight routes. The authors relate the apparently exponential (geometric) distribution of RHI above ice saturation (in stratospheric air also below saturation) to stochastic processes controlling water vapor, including the interaction of water molecules with cloud particles.

Statistical distribution laws of relative humidity around the $215 \mathrm{hPa}$ pressure level have also been deduced from satelliteborne Microwave Limb Sounder measurements (Spichtinger et al., 2002). Some features of the distributions of $S$ are similar to the MOZAIC results, but the measurements at the highest relative humidities (slightly above the homogeneous freezing nucleation limit of $\sim 165 \%$ at the lowest temperatures $\sim 185 \mathrm{~K}$ appearing in the Antarctic winter stratosphere) must be taken with care (Haag et al., 2003).

\subsection{Studying small-scale temperature fluctuations}

Our analysis in Sect. 3 and the previous work noted above used random samples of temperatures gathered on regional scales (horizontal scales of many $100 \mathrm{~km}$ ) to study the resulting distributions of RHI. On the mesoscale, down to scales of the order of $1 \mathrm{~km}$, buoyancy waves are known to produce rapid temperature oscillations in air parcels moving with the local wind. The mean amplitudes of the temperature fluctuations typically decrease with decreasing length scale.

We use our analytic tools to examine the distribution of RHI that result from mesoscale temperature fluctuations. Probability density functions of temperature fluctua- tion amplitudes derived from lower stratospheric measurements using the ER-2 research aircraft have been described by Bacmeister et al. (1999). The density functions can be fitted well with normal distributions within $\pm 1 \sigma$ of the peak, but exhibit non-Gaussian behavior in the wings, caused, in part, by intermittent large-amplitude wave events. The highamplitude tails become more pronounced as the length scale decreases; vice versa, Gaussians provide better fits to the overall distribution when the length scale increases.

In Fig. 2 of Bacmeister et al. (1999) such distributions are given for horizontal scales in the range $1-12.8 \mathrm{~km}$, which we can be aproximated according to

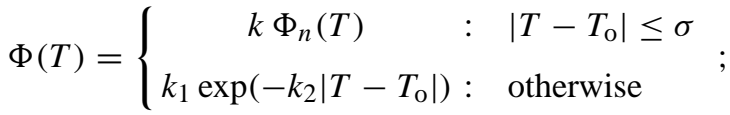

for the length scale of $12.8 \mathrm{~km}$, we use $\sigma=0.18 \mathrm{~K}$ and $k_{2}=6 \mathrm{~K}^{-1}$. The Gaussian $\Phi_{n}(T)$ is defined in Eq.(5). The constant $k_{1}$ follows from the requirement that both distributions in Eq.(18) should be equal at $\left|T-T_{\mathrm{o}}\right|=\sigma$, yielding the value

$k_{1}=k \frac{\exp \left(k_{2} \sigma\right)}{\sigma \sqrt{2 \pi \mathrm{e}}}$,

with $\mathrm{e}=\exp (1)$. The remaining normalization constant $k$ is determined by $\int_{-\infty}^{+\infty} \Phi d T=1$, leading to

$\frac{1}{k}=\operatorname{erf}\left(\frac{1}{\sqrt{2}}\right)+\frac{1}{k_{2} \sigma} \sqrt{\frac{2}{\pi \mathrm{e}}}$,

with the error function $\operatorname{erf}(x)=(2 / \sqrt{\pi}) \int_{0}^{x} d t \exp \left(-t^{2}\right)$. The distributions of RHI can then readily be computed (recall Sect. 3.1).

The distributions of $T$ and RHI are shown in Fig. 8 as solid curves, evaluated for $T_{0}=225 \mathrm{~K}$ and $\mathrm{RHI}_{\mathrm{o}}=100 \%$. Also 

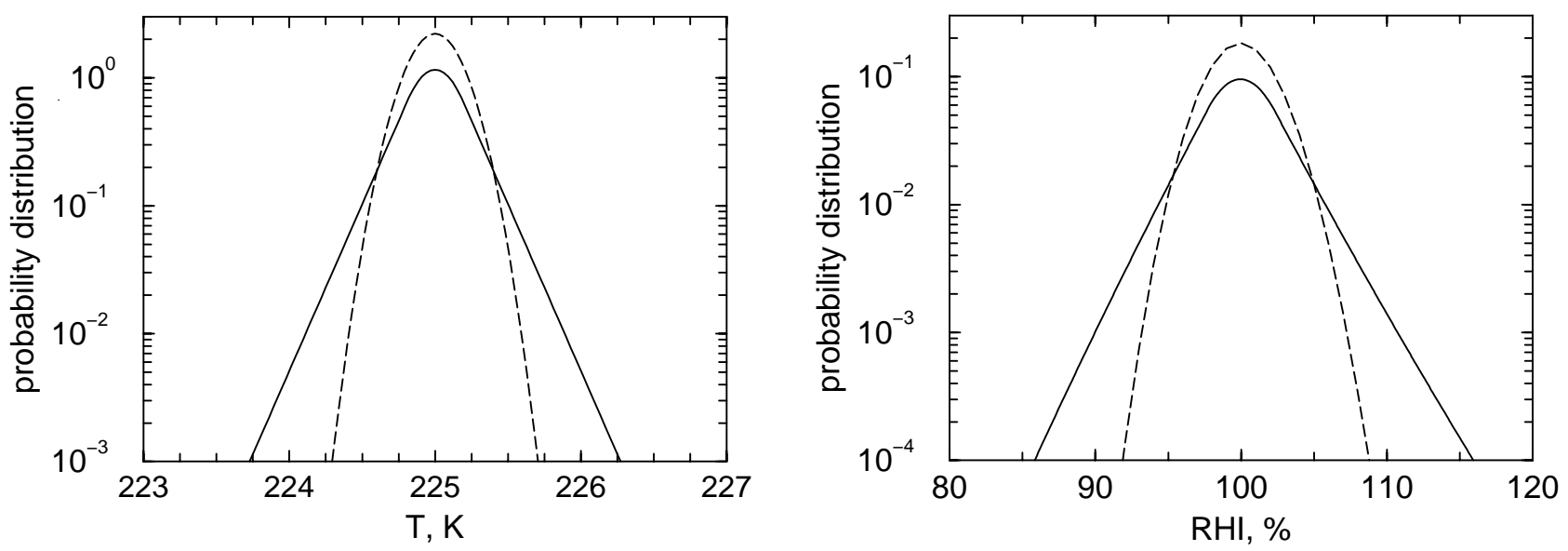

Fig. 8. Comparison between a temperature distribution arising from mesoscale temperature fluctuations with characteristic horiziontal scale of $12.8 \mathrm{~km}$ (Bacmeister et al., 1999) and the resulting distribution of RHI (solid curves, left and right panel, respectively) with corresponding solutions for an equivalent Gaussian temperature distribution (dashed curves).

shown are the results for a pure normal distribution of $T$ with $k=1$ and the same value of $\sigma$ and $T_{\mathrm{o}}$ (dashed curves). The high-amplitude wings of the temperature distribution including lower stratospheric mesoscale variability in cooling rates leads to much higher relative humidities within the icesupersaturated region than the equivalent Gaussian distribution. Variations of water mixing ratios are less important in this case, as $\chi$ will stay constant in an air parcel undergoing this type of vertical motions, apart from changes induced by slow diffusive mixing or cloud formation. The distribution of RHI with mesoscale temperature fluctuations is narrower than those shown in Fig. 2, where much larger standard deviations of $T$, corresponding to greater length scales, have been prescribed.

\subsection{Summarizing and discussing the main findings}

This work explicity states that changes in temperature are much more important in creating supersaturations with respect to the ice phase than changes in water vapor mixing ratios. Extending the previous work, we have quantified the link between variations of $T$ and the resulting distributions of RHI. Temperatures and water vapor mixing ratios from a regional airborne campaign and parameterized mesoscale temperature fluctuations are analyzed in support of this attempt.

We summarize the following issues from Sects. 3 and 4.1-4.3.

1. Variations of temperature over a wide range of atmospheric horizontal length scales is the key factor controlling the distributions of RHI, followed by variation of water vapor mixing ratios.

2. Exponential distribution laws used as limiting distributions for high values of RHI may serve as excellent approximations of atmospheric measurements of RHI.
3. Distributions of RHI in subsaturated regions may exhibit a multimodal structure, caused by a superposition of humidity modes originating from mixing of different air masses.

4. The interaction of water molecules with cloud particles is not required to explain details of the distribution of RHI below the cloud nucleation threshold.

We interpret these results in the light of previous work.

Point 1 emphasizes the fact that vertical air motions play a more important role in controlling the distributions of RHI than horizontal exchange processes that redistribute $\mathrm{H}_{2} \mathrm{O}$ mixing ratios. The Gaussian distributions of RHI evaluated at a fixed temperature, as shown in Fig. 3, are essentially equivalent to the distributions discussed by Gierens and Brinkop (2002) obtained with a two-dimensional, stochastic exchange model that simulates isothermal horizontal diffusion of water vapor. Typically, the slope towards high RHI of the Gaussian distribution falls off much more rapidly than the slope of the distribution generated by changes of $T$, compare Figs. 2 and 3 and recall Sect. 3.4. Further, the time scales over which horizontal exchange of air occurs driven by turbulent diffusion are rather long. A rough estimate is given by $L^{2} /(2 K) \simeq 9$ hours, with the spatial scale $L \simeq 1 \mathrm{~km}$ and the horizontal eddy diffusion coefficient $K \simeq 20 \mathrm{~m}^{2} \mathrm{~s}^{-1}$ (Schumann et al., 1995). In contrast, vertical air motions, especially those induced by mesoscale waves, are known to occur over a very wide range of temporal and spatial scales (Tabazadeh et al., 1996; Bacmeister et al., 1999; Gary, 2002; Kärcher and Ström, 2003). This explains why horizontal mixing of water vapor is much less efficient in producing high ice supersaturations and initiating the formation of cirrus clouds than vertical motions.

The distribution of RHI arising from mesoscale temperature fluctuations at horizontal scales of $\sim 10 \mathrm{~km}$ or less observed in the background lower stratosphere do not reach very far into the ice-supersaturated region, even if the mean 
relative humidity is close to ice saturation. In a real air parcel, a superposition of many gravity waves acting over a range of horizontal scales will determine the overall spread of the distributions of RHI. Thus, the width of the distribution of RHI shown in Fig. 8 may actually be underestimated if fluctuations arising from scales $>10 \mathrm{~km}$ are added. Together this indicates that ice nucleation in such temperature fluctuations may be restricted to regions where synoptic waves already lead to large-scale ice supersaturation and that the background fluctuations are then sufficient to push air parcel temperatures below the freezing threshold. In this regard it would be very helpful to learn more about temperature fluctuations in cirrus conditions in the upper troposphere and tropopause region.

Point 2 helps to elucidate the nature of the distribution laws of RHI. The discussion of the analytical results in Sects. 3.1 and 4.3 showed that the distributions may not exactly follow an exponential law at high RHI. However, we argue in the spirit of Gierens et al. (1999) that an exponential law is apparently a very good approximation in most cases (see Appendix for more details). The reason is expressed by the fact that the rates of dynamical and microphysical processes that control the appearance and disappearance of water molecules in the gas phase in a given volume of air are independent Poisson processes without memory (Gierens et al., 1999). In the Appendix, we derive the distributions of $T$ that lead to exact exponential distributions of RHI in the limit RHI $\gg \mathrm{RHI}_{0}$.

Point 3 states that if several $\chi$-distribution modes are superimposed at subsaturated values of RHI, the subsaturated region may exhibit a more complicated structure, possibly with multiple and overlapping maxima. The distribution functions of RHI below saturation from INCA, shown as blue curves in Fig. 7 or the cases discussed by Gierens et al. (1999, their Fig. 4), can be thought of as being composed of several humidity modes, each with different means and variances. The relative weight of each mode is determined by the respective volumes of air that mix. The slope of the distribution above saturation remains quasi-exponential as long as the mean values are sufficiently below ice saturation and the variances of $\chi$ are not very large. Similar arguments have been presented in Sect. 4.1 using the INCA data.

The possibility of several modes contributing to the distribution of RHI by mixing is of special relevance for the tropopause region, where the air masses exhibit both upper tropospheric and lower stratospheric character (e.g. high ozone amounts in rather humid air) (Kärcher and Solomon, 1999; Hoor et al., 2002; Fischer et al., 2003; Thornton et al., 2003).

Point 4 states that the properties of the distributions of RHI are controlled by the statistical properties of the controlling distributions of $T$. Cloud-related processes that possibly affect water vapor concentrations, as hypothesized by Gierens et al. (1999), are not required to explain the basic features of the distributions of RHI. We do not rule out potential indirect impacts of cloud processes on the distribution; for instance, condensation and subsequent transport of cloud water to lower altitudes by sedimentation or precipitation alters the distribution of $\chi$ and thus that of RHI. The impact of cirrus cloud formation on the distribution of RHI is discussed in a separate study (Haag et al., 2003), where it is explicitly shown that vertical air motion and the growth rates of ice crystals in cirrus determine the slope of the distributions of RHI.

\section{Conclusions}

The main results of this work have been discussed and summarized in Sect. 4.4. The simple tools provided here offer a straightforward explanation of how the basic features of distributions of relative humidity over ice in subsaturated and supersaturated regions come about and what their controlling factors are. The comparison with in situ measurements demonstrated the usefulness of our approach to interpret atmospheric observations of relative humidity on various horizontal length scales.

We hope that the ideas conveyed in the present study stimulate future work in this research area. Two important applications may concern improvements in predicting upper tropospheric relative humidity and cirrus clouds with largescale atmospheric models, a prerequisite for the proper treatment of cirrus clouds, and aid in the interpretation of measurements of temperature and relative humidity.

\section{Appendix : Exponential distribution laws of RHI}

In Sect. 4.4 (point 2) we have argued that an exponential law for $S$ approximates the distribution of $S$ very well towards high supersaturations. In some cases, an exponential distribution is found to fit measurements with an almost perfect correlation within a wide range of RHI values (Spichtinger et al., 2002), in other cases, the exponential distribution is a good fit only some $5-10 \%$ above ice saturation, see Fig. 7 or Gierens et al. (1999).

Here we answer the question: Which distribution of $T$ causes an exact exponential decrease of the distribution of $S$ towards high values of $S$ ? In the following, we ignore variations of $\chi$ and adiabatic pressure changes, as they do not significantly alter the results (recall point 1 in Sect. 4.4).

Combining Eqs. (3) and (6), we obtain

$\Psi(S)=\frac{\theta}{S \ln ^{2}(S / \alpha)} \Phi(S)$.

As the distribution of $S$ should decay exponentially above its maximum (approximately located at $S_{\mathrm{o}}$ ), we write

$\Psi(S) \stackrel{S \gg S_{0}}{\longrightarrow} C \exp (-S / \bar{S})$

where the parameters $\bar{S}$ and $C$ are constants. Using Eq. (2), the above expressions yield the asymptotic behavior of the distribution of $T$ for low temperatures,

$\Phi(T) \stackrel{T \ll \bar{T}}{\longrightarrow} C \frac{\omega}{\theta}\left(\frac{\theta}{T}\right)^{2} \exp \left[\frac{\theta}{T}-\exp \left(\frac{\theta}{T}-\frac{\theta}{\bar{T}}\right)\right]$, 

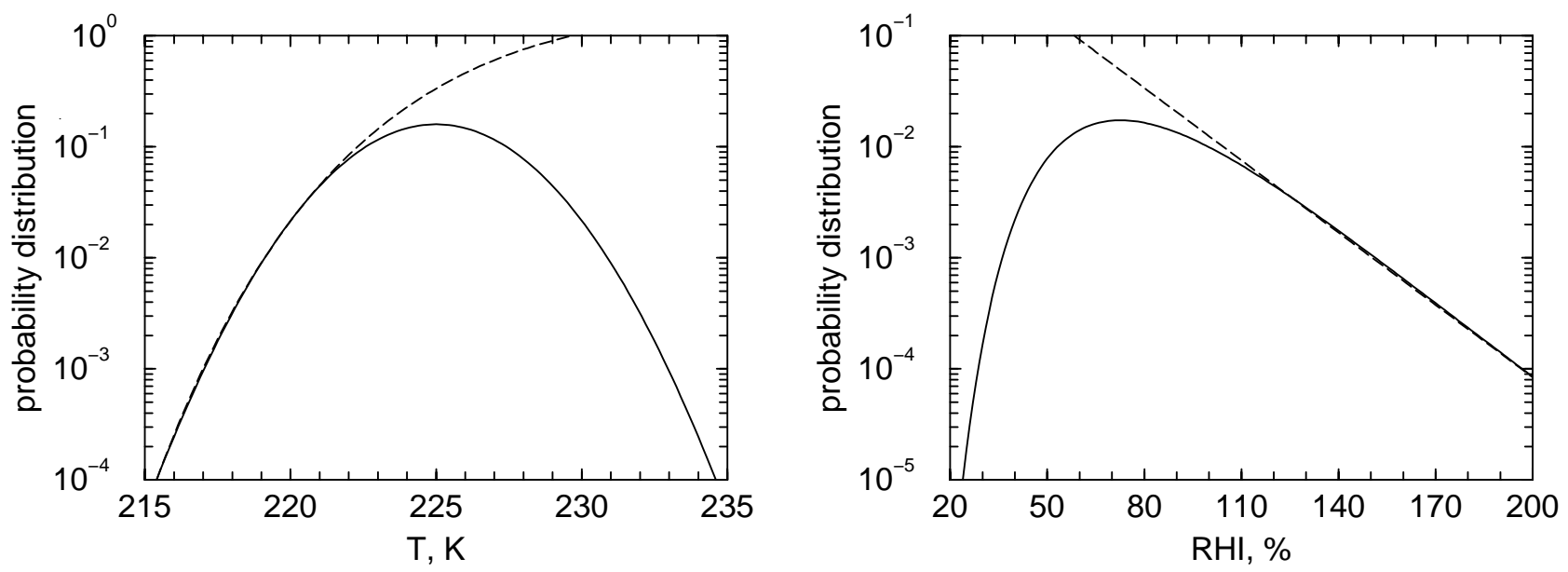

Fig. A1. Comparison between Gaussian temperature distribution and resulting quasi-exponential distribution of RHI (solid curves, left and right panel, respectively) with corresponding analytical solutions valid at low temperatures and high relative humidities (dashed curves) describing an exact asymptotic exponential decrease of the distribution of RHI.

with the parameter $\bar{T}$ at which $\Phi(T)$ reaches its maximum and $\omega=\bar{S} \exp (-\theta / \bar{T})$.

Figure A1 (left panel) depicts the normally distributed temperatures $\Phi_{n}(T)$ from Eq. (5) with $T_{0}=225 \mathrm{~K}$ and $\sigma=2.5 \mathrm{~K}$ (solid curve) along with the distribution Eq. (A3) with $C=1.85$ and $\bar{T}=237 \mathrm{~K}$ (dashed curve). The right panel depicts the resulting distributions of $S$ from Eq. (10) with $S_{0}=0.8$ (solid curve; the same distribution is plotted as a dashed red curve in Fig. 2) and from Eq. (A2) with $\bar{S}=0.2$ (a value consistent with the MOZAIC data).

The agreement between the exact Gaussian distribution for $T$ and the exact exponential distribution for $S$ with our analytic solutions Eqs. (A3) and (A2) is very remarkable. We may argue that much larger deviations from an exact exponential fall-off than those shown in Fig. A1 cannot be discerned from atmospheric measurements of RHI, because typical experimental uncertainties of RHI are some $\pm 5 \%$ or higher. Such deviations may be caused by additional variability due to adiabatic corrections and variations of $\chi$ (which have been ignored in the analytic solution) or by low temperature tails of the distributions of $T$ that do not exactly follow a Gaussian distribution or Eq. (A3), including those arising from small-scale temperature fluctuations as examined in Sect. 4.3.

Acknowledgements. We thank Klaus Gierens for comments on a draft version of this manuscript and Andrew Gettelman for a useful discussion. This research was conducted within the projects "Particles in the Upper Troposphere and Lower Stratosphere and Their Role in the Climate System" (PARTS) and "Interhemispheric Differences in Cirrus Properties From Anthropogenic Emissions" (INCA), funded by the European Commission, and contributes to the project "Particles and Cirrus Clouds" (PAZI) supported by the Helmholtz-Gemeinschaft Deutscher Forschungszentren (HGF).

Topical Editor O. Boucher thanks two referees for their help in evaluating this paper.

\section{References}

Bacmeister, J. T., Eckermann, S. D., Tsias, A., Carslaw, K. S., and Peter, Th.: Mesoscale temperature fluctuations induced by a spectrum of gravity waves: A comparison of parameterizations and their impact on stratospheric microphysics, J. Atmos. Sci., 56, 1913-1924, 1999.

Fischer, H., de Reus, M., Traub, M., Williams, J., Lelieveld, J., de Gouw, J., Warneke, C., Schlager, H., Minikin, A., Scheele, R., and Siegmund, P.: Deep convective injection of boundary layer air into the lowermost stratosphere at midlatitudes, Atmos. Chem. Phys., 3, 739-745, 2003.

Gary, B.: Mesoscale temperature fluctuations: An overview, http: //reductionism.net.seanic.net/bgary.mtp2/isentrop/index.html, 2002.

Gettelman, A., Randel, W. J., Wu, F., and Massie, S. T.: Transport of water vapor in the tropical tropopause layer, Geophys. Res. Lett., 29, doi:10.1029/2001GL013818, 2002.

Gierens, K. M., Schumann, U., Smit, H. G. J., Helten, M., and Zängl, G.: Determination of humidity and temperature fluctuations based on MOZAIC data and parameterisation of persistent contrail coverage for general circulation models, Ann. Geophysicae, 15, 1057-1066, 1997.

Gierens, K. M., Schumann, U., Helten, M., Smit, H., and Marenco, A.: A distribution law for relative humidity in the upper troposphere and lower stratosphere derived from three years of MOZAIC measurements, Ann. Geophysicae, 17, 1218-1226, 1999.

Gierens, K. M., and Brinkop, S.: A model for the horizontal exchange between ice-supersaturated regions and their surroundings, Theor. Appl. Climatol., 71, 129-140, 2002.

Haag, W., Kärcher, B., Ström, J., Minikin, A., Ovarlez, J., Lohmann, U., and Stohl, A.: Freezing thresholds and cirrus cloud formation mechanisms inferred from in situ measurements of relative humidity, Atmos. Chem. Phys., 3, 1791-1806, 2003.

Heymsfield, A. J., Miloshevich, L. M., Twohy, C., Sachse, G., and Oltmans, S.: Upper-tropospheric relative humidity observations and implications for cirrus ice nucleation, Geophys. Res. Lett., 25, 1343-1346, 1998. 
Hoor, P., Fischer, H., Lange, L., Lelieveld, J., and Brunner, D.: Seasonal variations of a mixing layer in the lowermost stratosphere as identified by the $\mathrm{CO}-\mathrm{O}_{3}$ correlation from in situ measurements, J. Geophys. Res., 107, doi:10.1029/2000JD000289, 2002.

Jeffreys, H., Theory of Probability, second edition, Oxford Univ. Press, 1948.

Jensen, E. J., Toon, O. B., Vay, S. A., Ovarlez, J., May, R., Bui, P., Twohy, C. H., Gandrud, B., Pueschel, R. F., and Schumann, U.: Prevalence of ice-supersaturated regions in the upper troposphere: Implications for optically thin ice cloud formation, J. Geophys. Res., 106, 17 253-17 266, 2001.

Kärcher, B., and Solomon, S.: On the composition and optical extinction of particles in the tropopause region, J. Geophys. Res., 104, 27 441-27 459, 1999.

Kärcher, B., and Ström, J.: The roles of dynamical variability and aerosols in cirrus cloud formation, Atmos. Chem. Phys., 3, 823838, 2003.

Koop, T., Luo, B. P., Tsias, A., and Peter, Th.: Water activity as the determinant for homogeneous ice nucleation in aqueous solutions, Nature, 406, 611-614, 2000.

Marti, J., and Mauersberger, K.: A survey and new measurements of ice vapor pressure at temperatures between 170 and $250 \mathrm{~K}$, Geophys. Res. Lett., 20, 363-366, 1993.

Murphy, D. M., Kelly, K. K., Tuck, A. F., Proffitt, M. H., and Kinne, S.: Ice saturation at the tropopause observed from the ER-2 aircraft, Geophys. Res. Lett., 17, 353-356, 1990.

Ovarlez, J., van Velthoven, P., Sachse, G., Vay, S., Schlager, H., and Ovarlez, H.: Comparisons of water vapor measurements from POLINAT 2 with ECMWF analyses in high-humidity conditions, J. Geophys. Res., 105, 3737-3744, 2000.

Ovarlez, J., Gayet, J.-F., Gierens, K., Ström, J., Ovarlez, H., Auriol, F., Busen, R., and Schumann, U.: Water vapour measurements inside cirrus clouds in Northern and Southern hemispheres during INCA, Geophys. Res. Lett., 29, 1813, doi:10.1029/2001GL014440, 2002.

Peter, Th.: Microphysics and heterogeneous chemistry of polar stratospheric clouds, Annu. Rev. Phys. Chem., 48, 785-822, 1997.
Press, W. H., Teukolsky, S. A., Vetterling, W. T., and Flannery, B. P., Numerical Recipes in Fortran 77, second edition, Cambridge Univ. Press, 1992.

Schumann, U., Konopka, P., Baumann, R., Busen, R., Gerz, T., Schlager, H., Schulte, P., and Volkert H.: Estimate of diffusion parameters of aircraft exhaust plumes near the tropopause from nitric oxide and turbulence measurements, J. Geophys. Res., 100, 14 147-14 162, 1995.

SPARC Assessment of Upper Tropospheric and Stratospheric Water Vapor, D. Kley and J. M. Russell III (Co-chairs), World Climate Research Program (WCRP), WCRP-113, World Meteorological Organization (WMO), WMO-TD No. 1043, Stratospheric Processes and Their Role in Climate (SPARC) Report No. 2, 233240, December, 2000.

Spichtinger, P., Gierens, K., and Read, W.: The statistical distribution law of relative humidity in the global tropopause region, Meteorol. Z., 11, 83-88, 2002.

Ström, J., Seifert, M., Kärcher, B., Minikin, A., Gayet, J.-F., Krejci, R., Petzold, A., Auriol, F., Haag, W., Busen, R., Schumann, U., and Hansson, H.-C.: Cirrus cloud occurrence as a function of ambient relative humidity: A comparison of observations from the Southern and Northern Hemisphere midlatitudes obtained during the INCA experiment, Atmos. Chem. Phys., 3, 1807-1816, 2003.

Tabazadeh, A., Toon, O. B., Gary, J., Bacmeister, J. T., and Schoeberl, M. R.: Observational constraints on the formation of type 1a polar stratospheric clouds, Geophys. Res. Lett., 23, 2109-2112, 1996.

Thornton, B. F., Toohey, D. W., Avallone, L. A., Harder, H., Martinez, M., Simpas, J. B., Brune, W. H., and Avery, M. A.: In situ observations of $\mathrm{ClO}$ near the winter polar tropopause, J. Geophys. Res., 108, 8333, doi:10.1029/2002JD002839, 2003.

Vay, S. A., Anderson, B. E., Jensen, E. J., Sachse, G. W., Ovarlez, J., Gregory, G. L., Nolf, S. R., Podolske, J. R., Slate, T. A., and Sorenson, C. E.: Tropospheric water vapor measurements over the North Atlantic during the Subsonic Assessment Ozone and Nitrogen Experiment (SONEX), J. Geophys. Res., 105, 3745$3755,2000$. 